Estudios Románicos, Volumen 30, 2021, pp. 219-230

ISSN: 0210-4911

eISSN: $1989-614 \mathrm{X}$

DOI: https://doi.org/10.6018/ER.438861

\title{
«FUITE » ET ÉCRITURE DU BIEN-ÊTRE EN ROMAN IVOIRIEN : ANALYSE DES SOLEILS DES INDÉPENDANCES D'AHMADOU KOUROUMA
} ("Escape" and writing of well-being in Ivorian novel: analysis of Les Soleils des indépendances by Ahmadou Kourouma)

\author{
Bidy Cyprien Bodo* \\ Université Félix Houphouët-Boigny
}

\begin{abstract}
The "escape" in question is in line with Henri Laborit's point of view. It's about escaping into the world of the imagination in which one can carve out a vast and rewarding territory in an anxiety-provoking context. Escaping into the imaginary world therefore appears to be a happy mechanism for avoiding environment and sociological alienation. The link that Laborit establishes between escape and well-being is manifested in Les Soleils des indépendances of Ahmadou Kourouma. This novel, under study, illustrates this theory. It's the place for the expression of the "eulogy of escape" to the analysis of the posture of two characters: Salimata and Fama through the deployment of what we call the "imagined-tranquilizing" that makes this reflection explicit.
\end{abstract}

Keywords: escape, imaginary, well-being, imagined-tranquilizing

Résumé : « La fuite » dont il est question s'inscrit dans l'optique d'Henri Laborit. Il s'agit de fuir dans le monde de l'imaginaire dans lequel on peut se tailler un vaste territoire gratifiant dans un contexte anxiogène. La fuite dans l'imaginaire apparaît par conséquent comme un mécanisme heureux d'évitement de l'aliénation environnementale, sociologique. Le lien que Laborit établit entre la fuite et le bien-être est manifeste dans Les Soleils des indépendances d'Ahmadou Kourouma. Cette œuvre, à l'étude, illustre cette théorie. Elle est le lieu de l'expression de «l'éloge de la fuite » à l'examen de la posture de deux personnages : Salimata et Fama à travers le déploiement de ce que nous appelons «l'imaginé-tranquillisant » que cette réflexion explicite.

Mots-clés : Fuite, imaginaire, bien-être, imaginé-tranquillisant.

\footnotetext{
*Adresse pour la correspondance : 22 Bp 1242 Abidjan 22, Côte d'Ivoire. (00225) 0707881194. [cyprienbodo@, yahoo.fr]
} 


\section{Introduction}

Ce texte est le fruit d'une réflexion présentée au forum international sur le « Bienêtre et les subjectivités », notamment dans le « Sud global » à l'Université de Tübingen en Allemagne ${ }^{1}$. L'objectif de cette rencontre était de débattre, de questionner la signification et l'opérationnalité de divers modèles et concepts du bien-être dans les domaines de la santé, de l'éducation, du travail, de la prospérité économique, de la participation communautaire, de la spiritualité, de la création culturelle et littéraire.

C'était pour nous l'opportunité de relire, sous l'angle du bien-être, Ahmadou Kourouma (1927-2003) au regard de son statut dans le champ littéraire africain qui en fait, selon Abdourahman Waberi dans la revue Notre Librairie ${ }^{\circ} 155-156^{2}$ (2004 : 68), un « colossal ». D'ailleurs, comme le conseillait Boniface Mongo-Mboussa (2002: 15), « il est grand temps de relire nos classiques. Non pas pour les opposer aux «modernes" Mais pour marquer une continuité et surtout pour mettre en exergue la modernité de certains classiques ». Et effectivement, la relecture d'un des classiques de Kourouma, Les Soleils des indépendances (1970), nous a permis de noter qu'à côté des questions intéressantes que sont, pour l'essentiel, la langue littéraire, le style, les mythes littéraires, le statut de la femme, la culture malinké, l'interculturalité, la problématique coloniale, la gestion des indépendances qui ont fait l'objet de nombreuses et pertinentes analyses ${ }^{3}$, se pose une, peu convoquée : la problématique moderne ou postmoderne du bien-être qui structure le parcours narratif de Salimata et de Fama dans cette œuvre.

Ce classique de l'auteur ivoirien Kourouma met en effet en scène Fama et Salimata confrontés au changement de paradigme culturel, politique, social, économique du fait de la colonisation puis des indépendances « ratées » avec leurs effets pervers que sont, pour Fama, la perte du pouvoir politique, de la puissance économique et culturelle, l'incarcération, la vie erratique. La situation de Salimata, son épouse, n'est guère enviable. En plus d'avoir été excisée puis violée, elle doit affronter la pauvreté, la stérilité et son lot d'humiliations, les difficultés familiales, etc. Fait notable, dans ces conditions picaresques, il se déploie chez ces deux personnages une voie très particulière, originale qui les aide à maintenir leur équilibre, un bien-être et qui n'a pas encore été soulignée par la critique ${ }^{4}$. C'est une voie intimiste, personnelle qui relève de la santé mentale ou du bien-être mental : il s'agit de la «fuite » conceptualisée par le chirurgien français Henri Laborit (1914-1995).

1 Le forum s'est déroulé sur un mois, du $1^{\text {er }}$ au 31 juillet 2020. Nous avons, pour l'essentiel, participé à l'atelier « Les lieux imaginaires du bien-être ».

2 Ce numéro spécial de cette revue est consacré à Kourouma et met l'accent sur son héritage littéraire.

3 Lire notamment, entre autres, Notre Librairie. Revue des littératures du Sud (2004), Dossier « Cahier spécial Ahmadou Kourouma : l'héritage. Identités littéraires ", n 155-156 juillet-décembre, Madeleine Borgomano, Ahmadou Kourouma, le " guerrier " griot (1998), Jean-Claude Nicolas, Comprendre Les Soleils des indépendances d'Ahmadou Kourouma (1985), Séwanou Dabla, Nouvelles écritures africaines. Romanciers de la Seconde Génération (1986), Makhily Gassama, La langue de Kourouma ou le français sous le soleil d'Afrique (1995).

4 La plupart des solutions du bien-être qui font actuellement l'objet des débats politiques et littéraires sont écologiques ou quantitatives (objectifs du développement durable). Sur la problématique écologique, le lecteur est renvoyé aux analyses de Jérôme Blanchet-Gravel sur «l'écoromantisme » dans son ouvrage La face cachée du multiculturalisme (2018:105-178) et celles de Baptiste Morizot sur la « solastalgie» dans la revue Critique $\mathrm{n}^{\circ} 860$ 861 (2019: 166-181). 
« Fuite » et écriture du bien-être en roman ivoirien :

Analyse des Soleils des indépendances d'Ahmadou Kourouma

La recherche des voies du bien-être structure les travaux de ce chirurgien. Ils portent, pour l'essentiel, sur les modalités de l'équilibre biologique pourvoyeur de gratification. Pour lui, il y a un lien entre l'équilibre biologique et le mieux-être. Dans cette logique, à partir de ses études sur la réaction organique aux agressions, il a mis en place un tranquillisant : la « chlorpromazine » à action psychotrope.

La prise de conscience des limites d'une approche strictement clinique, médicale l'a amené à ouvrir ses recherches à la biologie des comportements en situation sociale pour trouver d'autres voies. Il s'est ainsi intéressé aux sciences humaines, notamment à la psychologie, à la sociologie à partir desquelles il s'est forgé une conviction : « la fuite » comme la voie royale du bien-être. Elle est analysée dans son ouvrage au titre révélateur : Éloge de la fuite (1976). Le texte se positionne comme dithyrambique de «la fuite » dont il vante les mérites dans la construction du bien-être. D'où ce questionnement : qu'est-ce que la fuite? En quoi est-elle une modalité du bien-être? Comment les postures des personnages Salimata et Fama, dans Les Soleils des indépendances de Kourouma, illustrent-elles «l'éloge de la fuite »? De trois manières, fondamentalement, que l'étude met en exergue : la construction d'un autre-moi gratifiant, la fabrique d'un couple gratifiant et la victoire gratifiante de «la fin voulue » sur « la fin subie». Nous aborderons ces modalités de ce que nous appelons « l'imaginé-tranquillisant » après une approche définitionnelle du concept de la fuite laboritienne. Nous ne manquerons pas de proposer une interprétation de « la fuite » chez Kourouma avec l'objectif de montrer que cette posture, dans Les Soleils des indépendances, n'est pas l'expression d'un défaitisme. Elle pourrait se lire comme une manifestation d'un esprit décolonial à travers l'intranquillité sémantique qu'elle convoque et une distance critique du système politique postcolonial défaillant.

\section{Sémantique de la fuite laboritienne}

La fuite dont il est question avec Laborit est une démarche de la pensée. Elle est son activation à travers la mise en jeu de l'imaginaire, notamment en contexte anxiogène. Ainsi, pour Laborit,

Il y a plusieurs façons de fuir. Certains utilisent les drogues dites " psychotogènes ». D'autres la psychose. D'autres le suicide. Il y a peut-être une autre façon encore : fuir dans un monde qui n'est pas de ce monde, le monde de l'imaginaire. Dans ce monde, on risque peu d'être poursuivi. On peut s'y tailler un vaste territoire gratifiant, que certains diront narcissique. Peu importe, car dans le monde où règne le principe de réalité, la soumission et la révolte, la dominance et le conservatisme auront perdu pour le fuyard leur caractère anxiogène et ne seront plus considérés comme un jeu auquel on peut, sans crainte, participer de façon à se faire accepter comme «normal ». Dans ce monde de la réalité, il est possible de jouer jusqu'au bord de la rupture avec le groupe dominant en gardant intacte sa gratification imaginaire, la seule qui soit essentielle et hors d'atteinte des groupes sociaux. Ce comportement de fuite sera le seul à permettre de demeurer normal par rapport à soi-même. (Laborit $1976: 16,17)$ 
Le propos souligne le pouvoir de l'imaginaire, sa capacité à transformer le principe de réalité, d'ajouter de l'information, de transfigurer le monde qui entoure avec ses contraintes. L'imaginaire apparaît ainsi comme un mécanisme performant de fuite, d'évitement de l'aliénation environnementale, sociologique. En tant que lieu hors d'atteinte par l'autre, il se positionne comme lieu de liberté par excellence :

L'imaginaire s'apparente ainsi à une contrée d'exil où l'on trouve refuge lorsqu'il est impossible de trouver le bonheur parce que l'action gratifiante en réponse aux pulsions ne peut être satisfaite dans le conformisme socio-culturel. C'est lui qui crée le désir d'un monde qui n'est pas de ce monde. Y pénétrer, c'est choisir la meilleure part, celle qui ne sera point enlevée. (Laborit 1976 : 98)

En définitive, l'idée maîtresse chez Laborit est la possibilité qu'offre l'imaginaire d'échapper à la souffrance, à l'angoisse par la fuite et de construire en soi et pour soi un bien-être. Il est question, devant le «brutalisme ${ }^{5}$ » de la réalité (Mbembe, 2020), de fuir dans un imaginaire consolateur. C'est le jardin intérieur que l'on modèle à sa convenance comme en témoignent des éléments du parcours narratif de Salimata et Fama.

\section{Actualisation dans Les Soleils des indépendances : les modalités de l'imaginé-tranquillisant}

"L'imaginé-tranquillisant » exprime comment, dans une situation problématique, l'imaginé, la construction de la pensée, le lieu de l'imaginaire en somme contribue à atténuer, à dépasser le mal-être en fabriquant des conditions de bien-être. De la sorte, l'imaginé-tranquillisant est une expression de la fuite.

Dans Les Soleils des indépendances d'Ahmadou Kourouma, l'une des manifestations de l'imaginé-tranquillisant est la construction par Salimata d'un autre-moi gratifiant. Il s'agit pour ce personnage, par la puissance de l'imaginaire, de se mettre dans la peau d'une autre Salimata améliorée, meilleure. Il n'est pas ici question seulement de se projeter dans le moi idéal ou l'idéal du moi mais de le devenir et de le vivre. Il se produit alors une confusion-fusion entre soi et l'image imaginée de soi qui apaise parce qu'elle échappe, fusse-t-elle momentanée, à toute contingence crisogène comme en témoigne cet instant textuel de la vie de Salimata mis en évidence par un narrateur omniscient et donc en capacité de présenter les faits et d'instruire sur les effets, notamment les sentiments, les émotions de Salimata :

On se la montrait du doigt : Maudite beauté! une femme sans trou! une statuette! [...] Salimata vécut des mois et des années mais malheureusement sans enfant. Ce qui sied le plus à un ménage, le plus à une femme : l'enfant, la maternité qui sont plus que

5 Cette terminologie évoque chez Mbembe le processus de transformation de l'humain en valeur marchande. Elle est une réflexion sur la problématique du devenir-artificiel de l'homme avec ce que cela induirait comme violence épistémique et sociale. L'auteur ne manque pas de proposer des modalités pour y échapper. 
« Fuite » et écriture du bien-être en roman ivoirien :

Analyse des Soleils des indépendances d'Ahmadou Kourouma

les plus riches parures, plus que la plus éclatante beauté! À la femme sans maternité manque plus que la moitié de la féminité. Et les pensées de Salimata, tout son flux, appelèrent des bébés. Ses rêves débordaient de paniers grouillants de bébés, il en surgissait de partout. Elle les baignait, berçait et son cœur de dormeuse se gonflait d'une chaude joie au réveil. En plein jour et même en pleine rue, parfois elle entendait des cris de bébés, des pleurs de bébés. [...] Un matin, elle rinçait les calebasses; sous ses doigts elle sentit un bébé, un vrai bébé. Elle le baigna, il pleurait en gigotant. Elle le porta dans la chambre et ouvrit les yeux. Rien : une louche dure et cassante. [...] Une nuit, dans le lit, un bébé vint se coller à Salimata et se mit à téter, les succions ont brûlé les seins gauche et droit, elle le tâta, tout chaud, tout rond, tout doux. Elle alluma la lampe : envolé, transformé en mortier de cuisine. [...] Qu'importe qu'après que tout fût tombé, se fût envolé [...]. Salimata avait été heureuse; elle avait exulté; elle avait été enceinte, avait eu un ventre. (Kourouma $1970: 43,51,52$ )

Le propos du narrateur met en scène deux principes et leurs incidences sur le ressenti de Salimata. Le premier est le principe de réalité. Il présente Salimata sous les traits d'une femme stérile. Le narrateur en précise les effets à travers le jugement de valeur « malheureusement sans enfant» et le désignateur dépréciatif " femme à moitié de la féminité » qui témoignent d'une condamnation sociale et instruisent sur son mal-être. Face à ce réel problématique, Salimata trouve refuge dans la fuite laboritienne à travers l'activation de la pensée audacieuse, imaginative, créative, libre. Dans le territoire de son imaginaire, elle fabrique et devient une autre Salimata féconde, mère. Ainsi, la calebasse et la louche sont transformées en bébé et elle est également enceinte. Porté par la focalisation zéro, le narrateur ne manque pas de préciser l'évolution, le changement de ses sentiments une fois qu'elle habite le territoire de son imaginaire : "Salimata est heureuse ", " son cœur se gonflait d'une chaude joie ». Il se déploie de la sorte le principe du plaisir de l'imaginaire qui atténue celui de la réalité douloureuse. Pathos et éthos mettent dès lors en scène une Salimata améliorée, une « femme totale » dans le monde de son imaginaire. Le mal-être social y est vaincu et remplacé par le bien-être d'où l'idée d'imaginé-tranquillisant.

Cette autre Salimata témoigne de la capacité de l'imaginaire à transformer l'impuissance en puissance, l'incapacité en capacité et à créer un univers apaisant d'où l'éloge de la fuite chez Laborit. Le lieu de l'imaginaire se pose dès lors comme un espace de liberté, de possibilité de fabrique de bien-être auquel personne d'autre n'a accès et ne peut troubler. «Ce que nous appelons liberté, c'est la possibilité de réaliser des actes qui nous gratifient, de réaliser notre projet, sans nous heurter au projet de l'autre » (Laborit 1976 : 71). La liberté, dans le cas d'espèce, c'est, grâce à la fuite, l'indépendance, même relative, prise par Salimata devant les lois biologiques ou celles du déterminisme pour dire non à « l'autophagie» (Mbembe 2016 : 158). La fuite se lit de ce fait comme une soupape.

On objectera que, comme le montrent des éléments de l'extrait, la réalité finit par la rattraper par un mécanisme d'alternance enfant/calebasse/enfant/louche/enfant/mortier. L'enfant en effet finit par disparaître. Mais justement, la coprésence du réel et de l'imaginé, la tension réalité/dé-réalité (imaginaire), en l'occurrence, s'expliquent par la 
problématique du déni et du refoulement. Elles illustrent, dans le jeu de construction d'un bien-être, la différence entre le déni et le refoulement comme procédé de fuite. En effet, dans son ouvrage Figures du déni. En deçà du négatif, Bernard Penot instruit de cette différence. Contrairement au refoulement, dans le déni, « la représentation des choses ne s'y trouve pas soustraite en tant que telle. Elle n'est pas effacée comme image, mais c'est son sens qui s'avère indécidable » (Penot 2003 : 7). Cette lecture souligne que le refoulement nie l'existant quand le déni le problématise et s'autorise une distance critique et sémantique. Il (déni) se situe dans la méta-image et convoque la méta-lecture créatrice d'un méta-texte/corps du visuel. En conséquence, le déni, précise Penot (2003 : 7), affirme «l'existence du sujet comme acteur possible d'un jugement qui lui soit propre » instaurant par ce fait la dialectique de l'analogie et de la différence.

Le jeu de réalité/dé-réalité dans la scène analysée pourrait ainsi s'appréhender. Il témoigne de ce que d'une part, Salimata s'inscrit dans la dialectique laboritienne du « fuyard » (imaginaire) et de " l'installé » (la réalité) (Laborit 1976:49) qui la pose, à notre sens, comme une «fuyarde installée ». D'autre part, cette conflictualité illustre «l'agentivité » (Appadurai, 2013) de Salimata qui, à travers sa fuite portée par un « déni en deçà du négatif » se construit, dans la perspective de Simon Langlois (2014 : 389), un " bien-être subjectif » à côté d'un mal-être objectif. Comme le remarquait Virginie Affoué Kouassi (2004 : 51) dans son étude des femmes dans l'œuvre de Kourouma, Salimata est « douée de grandes capacités d'adaptation sous les soleils des indépendances ».

À l'autre-moi amélioré, Salimata associe, comme modalité de la fuite laboritienne, la fabrique d'un couple gratifiant à travers sa vision de Fama. En effet, le mari de Salimata apparaît dans la réalité du texte sous les traits d'un personnage dégradé, problématique (Lukacs, 1968). Pour preuves, Fama, « le prince », a pris les allures, à l'analyse de son jeu actantiel, d'une « hyène », d'un « vautour », d'un « charognard » (Kourouma 1970 : 11, 12). D'autre part, son parcours narratif est symptomatique d'une épopée dégradée. On peut souligner en effet qu'une histoire épique commence le plus souvent par un manque, se déploie par une quête et s'achève par la satisfaction du manque. Mais le sujet épique conquiert l'objet du manque à travers moult épreuves dites qualifiantes et obtient la reconnaissance, l'honneur au regard de ses actions glorifiantes, éclatantes. Pareillement, Les Soleils des indépendances commence par un manque. Le cousin Lacina avait évincé Fama de son trône dans le Horodougou. Sa destitution, qui introduit une nouvelle perturbation, relance la quête de Fama : reconquérir son pouvoir ce qui explique son voyage à Togobala. Mais une fois sur place, une épreuve qualifiante se présente à lui : l'opposition du parti unique incarné par le délégué. Comment le prince du Horodougou aborde-t-il cet obstacle? C'est à ce niveau que le mari de Salimata se distingue du personnage épique. Le narrateur rapporte : «Les partisans de Fama, et Fama, Diamourou et Balla, tous s'agenouillèrent, tous supplièrent. Le délégué se leva ébahi » (Kourouma 1970 : 136, 137). Ce parcours narratif de Fama se donne à lire comme une parodie du modèle épique traditionnel qui le présente sous l'angle d'un lâche, d'un picaro.

Tout autre est cependant la manière dont Salimata se représente son mari. Sa perception mentale est en retrait du principe de la réalité textuelle : 
« Fuite » et écriture du bien-être en roman ivoirien :

Analyse des Soleils des indépendances d'Ahmadou Kourouma

Un calme, une certaine indolence envahit Salimata. La paix du cœur. La lagune parut se rider sous les coups de tam-tams, des moteurs et des rythmes de cha-cha escamotés par le batelier. Mais le timbre de celui-ci rappelait Fama. Salimata se retourna, le chanteur était à demi caché par une passagère, mais la silhouette, autant qu'on pouvait la voir, rappelait aussi, confirmait : pas le Fama vautour vide, mais le jeune, le beau, que Salimata avait rejoint lorsqu'elle s'était échappée du village. [...] Le plus haut garçon du Horodougou, le plus noir, du noir brillant du charbon, les dents blanches, les gestes, la voix, les richesses d'un prince. [...] Et depuis, jamais [...] elle ne l'avait oublié [...] son Fama. Un Fama toujours unique. (Kourouma 1970 : 46, 47, 48)

Il apparaît nettement que le Fama de Salimata est une construction, une œuvre d'art. Il ne correspond pas à la réalité sociologique du texte que souligne le désignateur « le Fama vautour vide ». Son plaisir, sa joie de femme mariée, en somme son bien-être se trouve ainsi dans sa fuite dans l'imaginaire amoureux d'où la précision « son Fama » en opposition avec le Fama de la réalité. Toute tentative de Salimata de faire correspondre son image mentale de Fama à la réalité provoquerait la désillusion, le mal-être, ce à quoi elle se refuse d'ailleurs comme en atteste la précision narrative « son fama. Un Fama toujours unique ». Par conséquent, Salimata fait le choix de se fermer les yeux, de fuir le réel, de recréer, de fabriquer son univers amoureux car,

Le seul amour qui soit vraiment humain, c'est un amour imaginaire, c'est celui après lequel on court sa vie durant, qui trouve généralement son origine dans l'être aimé, mais qui n'en aura bientôt plus ni la taille, ni la forme palpable, ni la voix, pour devenir une véritable création, une image sans réalité. [...] L'amoureux est un artiste qui ne peut plus se passer de son modèle, un artiste qui se réjouit tant de son œuvre qu'il veut conserver la matière qui l'a engendrée. Supprimer l'œuvre, il ne reste plus qu'un homme et une femme; supprimer ceux-là, il n'y a plus d'œuvre. L'œuvre, quand elle a pris naissance, acquiert sa vie propre, une vie qui est du domaine de l'imaginaire, une vie qui ne vieillit pas, une vie en dehors du temps et qui a de plus en plus de peine à cohabiter avec l'être de chair. C'est pourquoi il ne peut y avoir d'amour heureux, si l'on veut à toute force identifier l'œuvre et le modèle. (Laborit $1976: 25,26)$

Applicable à souhait à Salimata, on conclura, au regard de sa posture laboritienne, que son amour pour Fama est devenu plus l'amour d'un concept que celui de la personne aimée. La fuite apparaît ici comme une pertinente stratégie de fabrique du bonheur ou du bien-être en amour.

La dernière manifestation de la fuite laboritienne que nous avons identifiée dans le roman de Kourouma est l'œuvre de Fama. Il s'agit de sa fuite par l'invention d'une finitude gratifiante. Elle a trait à sa posture dans les conditions de sa mort :

Les gardes frontaliers de la république de Nikina vinrent relever Fama qui avait été atteint. [...] Il était grièvement atteint à mort par le saurien. On coucha Fama dans une ambulance, direction Togobala du Horodougou. [...] On partait. Tu vas à Togobala, Togobala du Horodougou. Ah! voilà les jours espérés! La bâtardise 
balayée, la chefferie revenue, le Horodougou t'appartient, ton cortège de prince te suit, t'emporte. Ton cortège est doré. [...] Fama sur un coursier blanc qui galope, trotte, sautille et caracole. Il est comblé, il est superbe. [...] Tout s'arrange doux et calme, la douceur qui glisse, la femme qui console, et l'homme, et la rencontre d'un sous-bois frais et doux, et les sables menus et fins, et tout se fond et coule doucement et calmement, Fama coule, Fama avait fini [...]. Un Malinké était mort. (Kourouma $1970: 193,195,196)$

Cet extrait est porté par deux moments narratifs avec en toile de fond la technique de la fiction dans la fiction. Le premier informe des conditions objectives de la mort de Fama. On y apprend qu'il a été attaqué par un caïman, qu'il est grièvement blessé et agonisant dans une ambulance. Le second est celui de Fama dans une configuration du double littéraire. Il est le résultat de ce que David Le Breton (2004) nomme « les prolongements de soi ». Dans ce prolongement de soi, le récit de la situation de Fama s'oppose au premier. Il le réécrit, reconfigure les conditions de sa mort en nous conduisant dans le territoire de son imaginaire gratifiant, le lieu de sa fuite. Là-bas, Fama n'est pas couché dans une ambulance. Loin s'en faut! Il est le cavalier sur un cheval blanc métaphore de la pureté, de la puissance. Là-bas, il a vaincu la bâtardise, il a repris son trône, il a rétabli la dynastie des Doumbouya. Là-bas, il n'est plus ce personnage faible, mourant piteusement mais le digne, glorieux et victorieux chevalier africain. Il meurt ainsi en héros, en grand seigneur, en sauveur. Dans ce jeu de dédoublement, Fama, réfugié dans son imaginaire dans l'ambulance dans les derniers instants de sa vie, est le Don Quichotte africain. Il s'est fabriqué, par la fuite, une fin qui étouffe le récit de « la fin subie » ou « la fin honteuse » et dans laquelle il refuse de « se faire voler son image » (Gélinas 2011 : 75). En posant la situation finale du récit au cœur de «la fin voulue », de « la fin arrangée » ou inventée par Fama, le roman de Kourouma décline la victoire de l'imaginaire sur le réel. Et les adjectifs «calme », « doux », « superbe » et « frais », le participe passé « comblé » ainsi que le substantif « douceur » sans omettre les adverbes « doucement », "calmement » instruisent des effets de cette victoire sur le mourant : une mort gratifiante, un bien-être pendant la finitude, une « belle » mort grâce à la fuite.

\section{Interprétation de la fuite kouroumienne}

L'étude de la fuite chez Kourouma peut soulever des interrogations, notamment en rapport avec sa portée. Par exemple, les actes de fuite de Salimata et de Fama ci-dessus analysés ne seraient-ils pas une expression du défaitisme, de l'abandon, de la passivité devant la difficulté? Pour réponse à cette lecture, rappelons que la fuite laboritienne implique l'activation de la pensée créatrice, imaginative qui est, déjà, à l'opposé de la passivité. Leur posture est donc antinomique à « la défaite de la pensée » (Finkielkraut, 1987).

Par ailleurs, activer le champ de l'imaginaire pour se construire un havre de paix n'a pas signifié pour Salimata et fama rester passifs face aux contingences de leur vie. Pour preuves, Salimata a pris des dispositions sociales, posé des actes, lutté pour affronter 
et résoudre son problème de stérilité. Dans cette logique, elle a fréquenté « marabouts, sorciers », hôpital (Kourouma $1970: 51,53$ ). Elle a donc associé actes objectifs et imaginaire.

À un autre niveau, les modalités du recours à la fuite dans l'imaginaire par Fama et Salimata ont une valeur métaphorique, symbolique. Elles sont sémantiquement chargées. En effet, la distance sémantico-critique qu'ils ont prise avec les évènements pour les re-définir est un travail de re-conceptualisation fort instructif. Elle rappelle la « désobéissance épistémique » ou le « détachement épistémologique » (Mignolo 2015 : 27, 28) qu'instaure l'esprit décolonial et qui est manifeste dans cette méta-lecture de la colonisation et des indépendances faite par Fama :

Bâtard de bâtardise! Le soleil des indépendances maléfiques remplissait tout un côté du ciel, grillait, assoiffait l'univers. [...] Le soleil avait cesser de briller sur le quartier nègre pour se concentrer sur les blancs immeubles de la ville blanche. Damnation! bâtardise! les immeubles, les ponts, les routes de là-bas, tous bâtis par les doigts nègres, étaient habités et appartenaient à des toubabs. Les indépendances n’y pouvait rien! [...] Mais alors, qu'apportèrent les Indépendances à Fama? Rien que la carte d'identité nationale et celle du parti unique. Elles sont les morceaux du pauvre dans le partage et ont la sécheresse et la dureté de la chair du taureau. Rien à en tirer, rien à sucer. (Kourouma $1970: 11,20,25)$

La distance critique qu'induit le recours à la fuite laboritienne dans l'imaginaire et convoquée par Fama et Salimata peut être interprétée, dans le contexte actuel postcolonial, comme une invitation à l'esprit décolonial, à l'esprit de problématisation, à l'intranquillité sémantique devant le discours officiel dominant tant politique que socio-culturel que Laborit (1976 : 93) nomme « la caresse sociale ».

Enfin, Kourouma soutenait que la littérature africaine est « une littérature de mauvaise conscience » (Mongo-Mboussa, Préface, 2002 : 9). Dans cette logique, la fuite peut être lue comme un discours de «la mauvaise conscience » du système socio-politique postcolonial qui, à la lecture des Soleils des indépendances, apparaît incapable de répondre aux besoins des sujets africains postcoloniaux au point de les conduire à fuir dans l'imaginaire pour se construire un bien-être. Dans ce sens, la fuite est synonymique à une posture-révolte.

\section{Conclusion}

Jean-Claude Blachère (2004 : 19) note, à la lecture de Kourouma, que la tonalité générale souligne « l'impuissance des hommes à maîtriser leur destin ». En gardant à l'esprit cette remarque pertinente, une relecture des Soleils des indépendances nous a permis de mettre en exergue comment Fama et Salimata se jouent de cette impuissance en la transformant en puissance par le biais de la fuite laboritienne : l'activation de l'imaginaire, la fuite dans un monde qui n'est pas le monde objectif du texte, c'est-à-dire le monde de leur imaginaire. Dans ce monde parallèle ou monde dans le monde, ils se sont fabriqué une 
vie gratifiante, un destin voulu et non subi, un lieu de bien-être. C'est cet environnement socio-psychologique dans lequel ils se réfugient par la force de l'imaginaire que nous avons appelé « l'imaginé-tranquillisant ». C'est une sorte « d'écologie mentale », une voie de protection de la santé mentale de ces personnages. Elle est manifeste lorsque Salimata s'est construite pour son bien-être, en contradiction avec la réalité sociale textuelle, un autre-moi amélioré et heureux, un univers amoureux gratifiant et Fama une mort à la Roland $^{6}$, celle qui le peint comme l'incarnation de «l'esprit Kunta Kinté ${ }^{7} »($ Haley, 1977).

Un parallèle peut être établi entre la posture de Fama et Salimata et l'acte d'écriture ou la phase rédactionnelle chez Kourouma : "Je vivais dans leur monde, le monde créé » expliquait-il en répondant à une question posée par Tanella Boni sur le processus de fabrique de ses textes (Boni, 2004 : 78). Pareillement, dans Les Soleils des indépendances, Fama et Salimata vivent dans le monde qu'ils ont créé et dans lequel ils sont des personnages heureux. Dans l'illusion romanesque, ils apparaissent, dans le jeu du double littéraire, comme des " créés-créateurs ». En conséquence, par/grâce à la fuite dans l'imaginé-tranquillisant, ils ont pu goûter à la joie, au bien-être à l'intérieur d'une société de " bâtards de bâtardise », « de damnation » (Kourouma $1970: 11,27)$. Aucun appareil répressif exogène n'a pu et ne pouvait s'y opposer. Le lieu de leur imaginaire s'est posé comme un lieu de liberté par excellence. Y fuir est reconquérir la liberté :

Puisqu'il tient au cœur de l'individu de retrouver sa différence, de montrer qu'il est un être unique, ce qui est vrai, dans une société globale, ne peut-on lui dire que c'est dans l'expression de ce que sa pensée peut avoir de différent de celle des autres, et de semblable aussi, dans l'expression de ses constructions imaginaires en définitive qu'il pourra trouver le bonheur? (Laborit 1976 : 102)

Salimata et Fama répondent par l'affirmative et nous enseignent, à partir de la perspective du bien-être de Laborit, une pensée positive : tout le monde peut être heureux, en dehors des contingences ou capacités matérielles ${ }^{8}$, car chacun dispose, gratuitement, de ce lieu de la fuite : l'imaginaire. La fuite laboritienne opérationnelle dans le roman de Kourouma est une voie personnelle, intimiste du bien-être libre de toute pesanteur socio-politique.

Une autre portée de l'analyse a été soulignée à l'étude de l'esprit qui a prévalu à la fuite chez ces deux personnages : l'intranquillité sémantique, la désobéissance ou le détachement épistémique, la reconfiguration épistémique, la re-sémantisation qui rappellent l'esprit du sujet décolonial. Celui-ci (sujet décolonial) se positionne, à l'instar de Salimata et Fama,

$6 \quad$ Sur les conditions glorieuses de la mort de Roland, lire Jean Maurice, La Chanson de Roland, Paris, Puf, 1992.

7 Le lecteur est renvoyé à la posture du célèbre personnage d'origine africaine d'Alex Haley, Kunta Kinté, dans son texte Racines. Il incarne une des figures de la résistance.

8 Sur la question de l'importance des capacités matérielles dans la construction du bien-être, on renvoie le lecteur aux résultats des enquêtes de Simon Langlois (2014, p. 390, 391) qui soutiennent l'idée que « La grande majorité des enquêtes internationales conclut que le revenu des individus et des ménages est la principale variable explicative du sentiment de bien-être au plan transversal et microscopique. Plus le revenu disponible du ménage est élevé, plus le sentiment de bonheur et la satisfaction de ses membres à l'égard de la vie sont élevés. [...] Les taux moyens du bien-être se sont accrus avec la croissance des revenus ». 
« Fuite » et écriture du bien-être en roman ivoirien :

Analyse des Soleils des indépendances d'Ahmadou Kourouma

comme un « créé-créateur »: « un créé » par les contingences historiques, par exemple la colonisation, mais « un créateur » en tant que sujet participant, notamment épistémologiquement à la construction d'une modernité postcoloniale dans laquelle il se reconfigure comme sujet. Sous ce prisme, la fuite kouroumienne se lit comme une distance critique vis-à-vis des «monnè, outrages et défis » (Kourouma 1990) de l'histoire. Cette actualisation conforte le propos de Bonifasse Mongo-Mboussa qui recommandait de relire les classiques africains pour mettre en exergue leur modernité. En effet, Kourouma disait : « Un homme comme moi, du tiers-monde, a beaucoup à dire » (Borgomano, 2004 : 3). Le résultat est qu'il y a encore beaucoup à dire sur lui et son écriture sous des angles nouveaux.

\section{BIBLIOGRAPHIE}

APPADURAI, Arjun (2003) : Condition de l'homme global. Paris : Payot.

BLACHÈRE, Jean-Claude (2004) : « Monnè, outrages et défis : quelle histoire! », dans Notre Librairie. Revue des littératures du Sud, Dossier "Cahier spécial Ahmadou Kourouma : l'héritage. Identités littéraires », n¹55-156 de juillet-décembre : p. 17-21.

BLANCHET-GRAVEL, Jérôme (2018) : La face cachée du multiculturalisme. Paris : Éditions du Cerf.

BONI, Tanella (2004) : " Les " contre-dires » de l'Histoire », Entretien dans Notre Librairie. Revue des littératures du Sud, Dossier «Cahier spécial Ahmadou Kourouma : l’héritage. Identités littéraires », n¹55-156 de juillet-décembre : p. 77-80.

BORGOMANO, Madeleine (1998) : Ahmadou Kourouma, le " guerrier " griot. Paris : L'harmattan.

(2004), «Écrire, c'est répondre à un défi », dans Notre Librairie. Revue des littératures du Sud, Dossier «Cahier spécial Ahmadou Kourouma : l'héritage. Identités littéraires », n¹55-156 de juillet-décembre : p. 3-9.

DABLA, Séwanou (1986) : Nouvelles écritures africaines. Romanciers de la Seconde Génération. Paris : L'Harmattan.

FINKIELKRAUT, Alain (1987) : La défaite de la pensée. Paris : Gallimard.

GASSAMA, Makhily (1995) : La langue de Kourouma ou le français sous le soleil d'Afrique.: ACCT-Karthala.

GÉLINAS, Ariane (2011) : «Identité trouble : manifestations littéraires du double », dans Postures, Dossier « Vieillesse et passage du temps », $\mathrm{n}^{\circ} 14: \mathrm{p} .71-83$, disponible sur [http://revuepostures.com/fr/articles/gelinas-14 ;18/02/2021]

HALEY, Alex (1977) : Racines, traduction anglaise de Maud Sissung. Paris : Robert Laffont.

KOUASSI, Affoué Virginie (2004) : «Des femmes chez Ahmadou Kourouma », dans Notre Librairie. Revue des littératures du Sud, Dossier «Cahier spécial Ahmadou Kourouma : l'héritage. Identités littéraires », n¹55-156 de juillet-décembre : p. 50-54. 
KOUROUMA, Ahmadou (1970) : Les Soleils des indépendances. Paris : Seuil. (1990) : Monnè, outrages et défis. Paris : Seuil.

LABORIT, Henri (1976) : Éloge de la fuite. Paris : Robert Laffont.

LANGLOIS, Simon (2014) : " Bonheur, bien-être subjectif et sentiment de justice au Québec » dans L'Année sociologique, Vol. 64 : 389-420, [https://www.cairn. info/revue-1-annee-sociologique-2014-2-page-389-htm;17 /07/2020]

LE BRETON, David (2004) : « Les prolongements de soi », dans La mort et l'immortalité, encyclopédie des croyances, Frédéric Lenoir et Jean-Philippe de Tonnac (dir.). Paris : Bayard, p. 1549-1567.

LUKACS, Georg (1968) : La théorie du roman. Paris : Denoël.

MAURICE, Jean (1992) : La Chanson de Roland, Paris : Puf.

MBEMBE, Achille (2016) : Politiques de l'inimitié. Paris : La Découverte. (2020) : Brutalisme. Paris : La Découverte.

MIGNOLO, Walter (2015) : La désobéissance épistémique. Rhétorique de la modernité, logique de la colonialité et grammaire de la décolonialité. Bruxelles : Peter Lang.

MONGO-MBOUSSA, Boniface (2002) : Désir d’Afrique. Paris : Gallimard.

MORIZOT, Baptiste (2019) : « Ce mal du pays sans exil. Les affects du mauvais temps qui vient », dans Critique, $\mathrm{n}^{\circ} 860-861: 166-181$, [https://www.cairn.info/revuecritique-2019-1-page-166.htm ; 20/01/2021]

NICOLAS, Jean-Claude (1985) : Comprendre Les soleils des indépendances d'Ahmadou Kourouma. Issy les Moulineaux : Édition Saint Paul.

PENOT, Bernard (2003) : Figures du déni. En deçà du négatif. Toulouse : ERES.

WABERI, Abdourahman (2004) : "Colossal Kourouma », dans Notre Librairie. Revue des littératures du Sud, Dossier « Cahier spécial Ahmadou Kourouma : l'héritage. Identités littéraires ", n¹55-156 de juillet-décembre : p. 68-70.

\section{PROFIL ACADÉMIQUE ET PROFESSIONNEL}

Docteur de l'Université de Limoges, Bidy Cyprien Bodo est Maître de conférences à l'Université Félix Houphouët-Boigny de Cocody-Abidjan, Côte d'Ivoire. Il est membre de l'équipe de recherches Langues, Civilisation, Arts, Cinéma et Littérature : Littératures et Imaginaires des mondes (LACIL-LIM). Il s'intéresse à la forme picaresque du texte africain et aux études décoloniales. Il est l'auteur des ouvrages La décolonialité. Sémantique et pratiques textuelles (2020), La question du picaresque dans la littérature africaine. Théories et pratiques (2016) et co-auteur de L'imaginaire social : itinéraire sémantique, formes, actualité (2019) et de Les écritures de l'horreur en littératures africaines (2016).

Fecha de recepción : 13-11-2020

Fecha de aceptación : 05-04-2021 